\title{
On the fundamental group of a smooth projective surface with a finite group of automorphisms
}

\author{
By Rajendra Vasant GurJar and Bangere P. Purnaprajna
}

(Received Nov. 2, 2015)

(Revised Dec. 2, 2016)

\begin{abstract}
In this article we prove new results on fundamental groups for some classes of fibered smooth projective algebraic surfaces with a finite group of automorphisms. The methods actually compute the fundamental groups of the surfaces under study upto finite index. The corollaries include an affirmative answer to Shafarevich conjecture on holomorphic convexity, Nori's well-known question on fundamental groups and free abelianness of second homotopy groups for these surfaces. We also prove a theorem that bounds the multiplicity of the multiple fibers of a fibration for any algebraic surface with a finite group of automorphisms $G$ in terms of the multiplicities of the induced fibration on $X / G$. If $X / G$ is a $\mathbb{P}^{1}$-fibration, we show that the multiplicity actually divides $|G|$. This theorem on multiplicity, which is of independent interest, plays an important role in our theorems.
\end{abstract}

\section{Introduction.}

In this paper by a surface, unless otherwise stated, we mean a projective algebraic surface.

A smooth surface $X$ is said to be an anticanonical surface if $X$ is rational and there is an effective divisor linearly equivalent to $\left|-K_{X}\right|$.

Let $X$ be a smooth projective surface/C and $G$ a finite group of automorphisms of $X$. Let $Y$ be the quotient surface $X / G$. One of the central concerns of this paper is the relationship between $\pi_{1}(X)$ and $\pi_{1}(X / G)$, when $X / G$ is either a $\mathbb{P}^{1}$-fibration over an arbitrary curve, an anticanonical rational surface or a minimal surface of Kodaira dimension zero. The surfaces of general type that are finite covers of such surfaces, especially of $\mathbb{P}^{1}$-fibrations, occur frequently in a range of questions relating to the geometry of surfaces of general type; topology, classification problems, in the study of linear series of surfaces and threefolds such as Calabi-Yau, to name a few. As applications of the work on fundamental groups, we prove Shafarevich conjecture on holomorphic convexity of the universal cover for these surfaces. Other corollaries of interest include an affirmative answer to a conjecture of Nori on fundamental groups and free abelianness of second homotopy groups for these surfaces.

Kodaira classified the singular fibers of an elliptic fibration. In this case the multiple fibers occur with arbitrarily large multiplicities. For fibrations of arbitrary genus the

2010 Mathematics Subject Classification. Primary 14F35; Secondary 14J29, 14L30, 14H30, 14D05, $14 \mathrm{~J} 50$.

Key Words and Phrases. surfaces of general type, fibrations, fundamental groups of algebraic surfaces, Shafarevich conjecture, holomorphic convexity, finite group actions on varieties, base change.

The research was partially supported by NSF grant 1206434 . 
number of types of singular fibers are enormous and such a classification is not tenable. Qualitative statements about the nature of multiple fibers are a reasonable question to ask. In this article we prove a theorem that bounds the multiplicities of multiple fibers for any fibered surface with a finite group of automorphisms. When $X / G$ is a $\mathbb{P}^{1}$-fibration, we obtain a much stronger statement. These results are of independent interest. However these results play a crucial role here and are used towards proof of the main theorem on fundamental groups.

The fundamental group of a smooth projective surface $X$ which is not of general type is well understood. Hence we sometimes assume that $X$ is of general type. In this case $\operatorname{Aut}(X)$ is well-known to be finite. This explains our assumption about action of a finite group $G$ of automorphism on $X$.

We first concentrate on the main results of this paper concerning fundamental groups. Before we state the theorem, some definitions are in order:

We say that a fibration $f: Y \rightarrow B$ on a smooth projective surface onto a smooth curve $B$ becomes a $C^{\infty}$-fiber bundle after a base change if there is a finite morphism $C \rightarrow B$ from a smooth curve $C$ onto $B$ such that the normalized fiber product $\overline{Y \times{ }_{B} C}$ is a $C^{\infty}$-fiber bundle over $C$.

Note. From now on we make a blanket assumption that all the surfaces in this article have the property that they do not become $C^{\infty}$-fiber bundle after a base change. We call this the fibration hypothesis $(\mathrm{FiH})$. This is needed since the Main Theorem below is not true for $C^{\infty}$-fiber bundles.

THEOREM 1 (Main Theorem). Let $X$ be a smooth projective surface with an action of a finite group $G$ such that $|G|$ is a product of district primes. Assume that all the isotropy subgroups of points are normal in $G$ and that $X$ satisfies $(F i H)$. Let $X / G$ admit a minimal $\mathbb{P}^{1}$-fibration $X / G \rightarrow C$. Then the image of the map $\pi_{1}(F) \rightarrow \pi_{1}(X)$ is finite, where $F$ is a general fiber of the map $X \rightarrow D$ induced from $X / G \rightarrow C$. In particular, the homomorphism $\pi_{1}(X) \rightarrow \pi_{1}(X / G)$ has kernel and cokernel both finite.

Corollary FOR $Z_{p}$. In particular, if $G=Z_{p}$, the theorem holds.

This result greatly generalizes the result in $[\mathbf{3}]$. In $[\mathbf{3}]$ we considered the case when $X$ admits a hyperelliptic fibration $f: X \rightarrow D$ such that all the fibers of $f$ are irreducible and $f$ has a "genuine" singular fiber. There is a natural action of the group $G=\mathbb{Z} /(2)$ on $X$ such that $X / G$ has a $\mathbb{P}^{1}$-fibration $X / G \rightarrow C$. We proved that in this case the image $\pi_{1}(F) \rightarrow \pi_{1}(X)$ is finite, where $F$ is a general fiber of $f$.

An interesting corollary of the Main Theorem is the Shafarevich conjecture on holomorphic convexity. Before we introduce the result, we recall a definition.

A complex manifold $U$ is holomorphically convex if for any sequence of points $\left\{x_{n}\right\}$ in $U$ without a limit point there is a holomorphic function $f$ on $U$ such that the sequence $\left|f\left(x_{n}\right)\right|$ is unbounded.

Shafarevich ConjeCture (SC). Let $X$ be a smooth projective variety/C. Then the universal covering space $\widetilde{X}$ of $X$ is holomorphically convex.

As a corollary of the Main Theorem we have: 
Corollary (SC). Let $X$ be as in the Main Theorem. Then the Shafarevich conjecture holds for $X$.

Another interesting corollary of the main result of this paper is an affirmative answer to a question of Madhav Nori (see [7]) for these surfaces we are dealing with in this paper.

Corollary. Let $X \rightarrow C$ satisfy the same hypothesis as in the Main Theorem. Let $B$ be an irreducible curve on $X$ such that $B^{2}>0$. Let $\bar{B}$ be the normalization of $B$ in its function field. Then the normal subgroup generated by the image of the natural homomorphism $\pi_{1}(\bar{B}) \rightarrow \pi_{1}(X)$ has finite index in $\pi_{1}(X)$.

In particular, if $X$ contains an irreducible rational curve $B$ with $B^{2}>0$ then $\pi_{1}(X)$ is finite.

In Section 3 we prove a factorization theorem. Let $X$ be a smooth surface of general type with a finite group of automorphisms $G$ satisfying the hypothesis in the Main Theorem above. Let $X / G$ have a $\mathbb{P}^{1}$-fibration over a curve $C$. The factorization theorem states that we can factor the quotient map $g: X \rightarrow X / G$ into two maps, $g_{H}: X \rightarrow X / G_{H}$ and $g_{V}: X / G_{H} \rightarrow X / G$ such that the map $g_{V}$ has only vertical branching and $g_{H}$ has only horizontal branching. It will be shown that $X / G_{H}$ is again a $\mathbb{P}^{1}$-fibration over a different curve $C^{\prime}$. In other words, without loss of generality, after a suitable factorization we can always assume in the context of the paper, that the finite map $g$ is branched only along horizontal components. So from now we have the following:

BAsic Setup. All our branching curves will be assumed to be horizontal. Please note, as stated above, this is not an extra hypothesis, but it follows from the hypothesis in the main theorem above, proof of the factorization results are in Section 3.

There are substantial technical difficulties in the proof for the general case that were absent in [3]. The first and foremost are the singularities that occur in the quotient $X / G$ for a more general finite group $G$. They are, naturally, far worse than the singularities under $\mathbb{Z} /(2)$ quotient. We first study the singularities of the quotient and classify them in the context of this paper (Section 3 ). In the case of $\mathbb{Z} /(2)$, the multiplicity of the fibers was 2 in the case of fibers of odd genus and there were no multiple fibers at all in the case of even genus. This issue of singularities of $X / G$ and multiple fibers of $X$ is inescapable and has to be dealt with. Also, for $\mathbb{Z} /(2)$, we do not have to reckon with the mischief played by isotropy subgroups to contend with. In the more general case dealt with here, they are unavoidable.

In Section 3, we prove the analogue of the Main Theorem for a smooth surface of general type $X$ with an action of any cyclic group $G$, such that $X / G$ is a smooth minimal $\mathbb{P}^{1}$-fibration (see Theorem $1^{\prime}$ ).

One of the crucial steps in the proof involves a base change to get rid of multiple fibers. It is an important step in the proof to bound the multiplicities of the fibers, if indeed there is such a bound. We now state a general theorem in this article that bounds the multiplicity of the singular fibration of a surface of general type with a finite group of automorphisms. If $X / G$ is a $\mathbb{P}^{1}$-fibration, then there is a much stronger result, as indicated in (ii) below. 
Theorem 2 (Multiplicities of fibers and $|G|$ ). Let $f: X \rightarrow C$ be a fibration on a smooth projective surface with irreducible general fiber. Let $G$ be a finite group of automorphisms of $X$ which permutes the fibers of $f$. Let $Y:=X / G$ with induced fibration $Y \rightarrow C / G$. Then we have the following assertions:

(i) For a fiber $G_{0}$ if $f$, let $F_{0}$ be the corresponding fiber of $Y \rightarrow C / G$. Then the multiplicity of $G_{0}$ is at most equal to the product of $|G|$ and the multiplicity of $F_{0}$.

(ii) If $g: Y \rightarrow C / G$ is a $\mathbb{P}^{1}$-fibration such that all the fibers of this map are irreducible then the multiplicity of $F_{0}$ divides $|G|$, where $F_{0}$ is an arbitrary fiber of $g: Y \rightarrow C / G$ (see Theorem 3 in Section 5).

We now state a result when $X / G$ is a smooth surface of lower Kodaira dimension that is not a $\mathbb{P}^{1}$-fibration.

THEOREM 4. Let a group $G$ of prime order act on a smooth projective surface $X$ of general type. Let $X / G$ be either a smooth rational surface with an effective anticanonical divisor or a minimal surface of Kodaira dimension zero. Then $\pi_{1}(X)$ is isomorphic to $\pi_{1}(X / G)$. In particular, if $X / G$ is either an anticanonical rational surface or a $\mathrm{K} 3$ surface, then $X$ is simply-connected.

The proof of this uses different arguments than those for Theorem 1 .

We now make some remarks that makes it clear why the hypothesis mentioned in the theorems are necessary.

\section{REMARKS.}

(1) In the proof of (ii) we crucially use some results of Sakai [6] on minimal ruled fibrations. Theorem 2 helps in mitigating the effects of singularities to some extent. Our theorems proved are optimal in several ways.

(2) In the theorem we have assumed that $X / G$ is a $\mathbb{P}^{1}$-fibration. Example 2 in Section 7 shows that if $X / G$ is not a $\mathbb{P}^{1}$-fibration, then the image of the homomorphism $\pi_{1}(F) \rightarrow \pi_{1}(X)$ can be infinite. In the example, the fibers of $X / G$ are even irreducible and smooth, but are of higher genus.

The construction of families of genus-3 fibrations in [4] shows that Theorem 1 is the best possible in various ways. Some examples are also given in $[\mathbf{9}$, Section 3$]$ of a surface of general type fibered by genus- 2 curves.

(3) In particular, the examples in [4] show that if the group is not a product of distinct primes the result is not true. The examples in [4] are quadruple Galois covers of surfaces of minimal degree, which are minimal $\mathbb{P}^{1}$-fibrations.

(4) One of the technical results in this paper involves a nice statement about factorization of the finite map in the Main Theorem into two maps, first of which has only vertical fibers as the branch divisor, and the second has only horizontal components in the branch divisor. This is possible because $|G|$ is product of distinct primes. If the order of $G$ is not a product of distinct primes, we have a counterexample to the Main Theorem again coming from genus three fibrations [4]. 
(5) There is the classical example of $S_{3}$ action on a surface, where not all isotropy subgroups are normal. This results in a situation where the branch divisors have higher order singularities and are no longer normal crossing divisors. The statements on fundamental groups are generally untenable without an assumption like this; an illustration is provided by the failure of Zariski's conjecture for higher order singularities that are not nodes.

Acknowledgements. We are very thankful to Madhav Nori for pointing out a mistake in our initial work. He also showed us an example which was very useful in correctly formulating our results. We are very thankful to the referees for reading the manuscript critically, pointing out some inaccuracies and making helpful suggestions to correct some proofs. We are thankful to Liana Sega for arranging for us a room to work in the campus of University of Missouri, Kansas City. We are thankful to Vinay Wagh for helping us with drawing many of the dual graphs in this paper.

\section{Preliminaries.}

All our varieties will be defined over the field of complex numbers $\mathbb{C}$. We will freely use elementary topological results in our proofs.

A smooth projective rational curve $A$ on a smooth projective surface $Z$ is called a $(-n)$-curve if $n>0$ and $A^{2}=-n$.

Let $\pi: X \rightarrow C$ be a surjective morphism from a smooth projective surface onto a smooth curve such that a general fiber of $\pi$ is irreducible. Recall that if $G_{0}:=\sum a_{i} G_{i}$ is a scheme-theoretic fiber of $\pi$ with $G_{i}$ irreducible components of $G_{0}$ then the greatest common divisor of the $a_{i}$ s is called the multiplicity of $G_{0}$. This definition makes sense even if $X$ is only normal. We denote it by $\operatorname{mult}\left(G_{0}\right)$.

If $H$ is a finite group of automorphisms of a smooth projective variety $X$ then in general $X / H$ is singular. Similarly, if $Y$ is a smooth projective variety and $X \rightarrow Y$ is a finite Galois covering then in general $X$ is singular.

For a non-cyclic quotient singular point $(Y, p)$, the three linear branches in the dual graph of the minimal resolution have absolute values of the determinants $d_{1}, d_{2}, d_{3}$ which satisfy $\sum\left(1 / d_{i}\right)>1$. Hence $\left\{d_{1}, d_{2}, d_{3}\right\}$ is one of the Platonic triples $\{2,2, n\},\{2,3,3\}$, $\{2,3,4\},\{2,3,5\}$. If two of the three determinants $d_{1}, d_{2}, d_{3}$ are equal then we have $\{2,2, n\},\{2,3,3\}$ as the only possibilities. This applies to the dual graph of a singular fiber $\widetilde{F}_{0}$ in Proposition 1 . (Note that the notorious triple $\{2,3,5\}$ does not appear in this case.)

This description of the singular fiber is used repeatedly in Section 5 .

We state some well-known results about ramification and branch curves for finite morphisms between normal algebraic surfaces.

Let $f: W \rightarrow Z$ be a finite surjective morphism between normal algebraic surfaces $W, Z$. A point $x \in W$ is ramified for $f$ if $f$ is locally analytically not a biholomorphic map near $x$. We say that the point $f(x)$ is a branch point for $f$ if $x$ is a ramified point for $f$. The set of ramified (resp. branch) points is a union of (closed) irreducible curves $R_{i}$, called ramified curves, and finitely many isolated points. Similar statement is true for the set of branch points. 


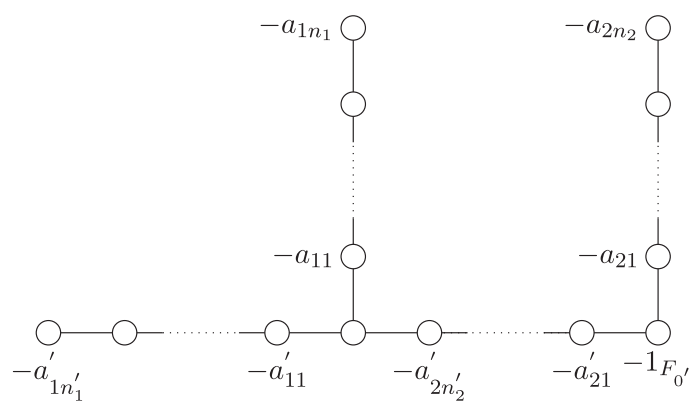

Figure 1.

The proof of the next result is easy so we omit it.

LEMMA 1. With the above notation, for a general point $x$ in a ramified curve $R_{i}$ we can choose local holomorphic coordinates $\left(w_{1}, w_{2}\right)$ near $x$ and $\left(z_{1}, z_{2}\right)$ near $f(x)$ such that $z_{1}=w_{1}^{m}, z_{2}=w_{2}$ and $R_{i}=\left\{w_{1}=0\right\}$ with $m>1$. If $f$ is a Galois morphism with Galois group $G$ then $G_{x} \cong \mathbb{Z} /(m)$.

The integer $m$ is the ramification index of $R_{i}$ over $B_{i}:=f\left(R_{i}\right)$. In particular, for a ramified curve $R_{i}$ the isotropy group $G_{x}$ is same for all but finitely many points $x \in R_{i}$. We denote this group by $G_{R_{i}}$. If $f_{1}: W \rightarrow Z, f_{2}: Z \rightarrow T$ are finite surjective morphisms and $R_{i}$ a ramified curve for $f_{1}$ then $R_{i}$ is also ramified for $f_{2} \circ f_{1}$. The ramification index of $R_{i}$ over $f_{2} \circ f_{1}\left(R_{i}\right)$ is the product of the ramification indices of $R_{i}$ over $f_{1}\left(R_{i}\right)$ and $f_{1}\left(R_{i}\right)$ over $f_{2}\left(f_{1}\left(R_{i}\right)\right)$. This follows easily from the above local description of $f_{i}$.

It is well-known that if a finite group $G$ acts on a normal variety $W$ and the isotropy group $G_{x}$ of a point $x \in W$ has order $>1$ then with respect to suitable local holomorphic coordinates $w_{1}, \ldots, w_{n}$ on $W$ at $x$ (where $n$ is the embedding dimension of the local ring of $W$ at $x)$ the action of $G_{x}$ is linear, i.e. $G_{x} \subset G L(n, \mathbb{C})$. In particular, if $x$ is a smooth point of a surface $W$ then $n=2$. Assume further that $G_{x}$ is abelian (this happens, for example, if $x$ is a general point of a ramified curve $R_{i}$ ). Then we can assume that the action of $G_{x}$ is diagonal with respect to $w_{1}, w_{2}$.

An element $\sigma \in G L(2, \mathbb{C})$ of finite order is called a pseudo-reflection if rank $(\sigma-I) \leq$ 1 . In this case, at most one eigenvalues of $\sigma$ is not equal to 1 . With respect to a suitable basis of $\mathbb{C}^{2}, \sigma$ is the diagonal matrix with eigenvalues $1, \omega$ for a suitable root of unity $\omega$. Suppose that $f: W \rightarrow Z$ is a finite surjective Galois morphism of algebraic surfaces with Galois group $G$. Assume that $x$ is a smooth points of $W$. Then by the ShephardTodd-Chevalley Theorem $f(x)$ is a smooth point of $Z$ if and only if $G_{x}$ is generated by pseudo-reflections. Any pseudo-reflection $\sigma$ as above fixes the curve $\left\{w_{2}=0\right\}$ pointwise. Here $\sigma\left(w_{1}, w_{2}\right)=\left(w_{1}, \omega w_{2}\right)$ with respect to suitable local holomorphic coordinates $w_{1}, w_{2}$ on $W$ at $x$. This applies for a general point $x$ lying on a ramified curve $R_{i}$ in $W$.

Assume that $f: W \rightarrow Z$ is a finite surjective morphism as above and $x$ is a smooth point of $W$ with $G_{x}$ abelian. Then as above, the action of $G_{x}$ is diagonal with respect to local coordinates $w_{1}, w_{2}$ near $x$. From this we see easily that the only curves passing through $x$ which are ramified are given by $\left\{w_{i}=0\right\}$ for some $i$. This implies that the 
branch curves passing through $x$ are smooth at $x$ and intersect transversally at $x$. An important consequence of these observations is the following result which will be useful later.

Lemma 2. Let $f: W \rightarrow Z$ be a finite surjective morphism as above. Assume that $W$ is smooth, $Z$ is normal and $G$ is abelian. Then the union of ramified curves in $W$ is a simple normal crossing divisor $R$.

Suppose that $G$ is finite and acts on a smooth surface $W$. Since the action of $G$ at a fixed point of $G$ is linear with respect to suitable local coordinates the set of fixed points of $G$ in a neighborhood of $x$ is a smooth (locally closed) subvariety of $W$. This implies that the set of fixed points in $W$, denoted by $W^{G}$, is a smooth closed subvariety of $W$. If the action of $G$ is faithful then $W^{G}$ is a proper subvariety of $W$.

REMARK. In contrast to the above the set of points in $W$ which have a non-trivial isotropy group may not be smooth. In Section 7 we will mention an example of this phenomenon.

The next result will be needed crucially later in the proofs.

LEMMA 3. Let a finite group $G$ act faithfully on a smooth surface $W$ such that the quotient $Z:=W / G$ is smooth. Assume that for any point $x \in W$ the isotropy subgroup $G_{x}$ is normal in $G$. Then $G_{x}$ is abelian for every $x \in W$.

Proof. As mentioned above $G_{x}$ is generated by pseudo-reflections $\sigma_{i}$ for $i=$ $1,2, \ldots, n$. Let $L_{i}$ be the hyperplane passing through $x$ which is fixed pointwise by $\sigma_{i}$. Now $\left(\sigma_{i}\right)$ is the isotropy subgroup of a general point of $L_{i}$. Hence $\left(\sigma_{i}\right)$ is a normal subgroup of $G$ for each $i$.

We claim that for $i \neq j$ the intersection $\left(\sigma_{i}\right) \cap\left(\sigma_{j}\right)$ is trivial. Suppose that this is not true. The set of points fixed by $\left(\sigma_{i}\right) \cap\left(\sigma_{j}\right)$ is a smooth closed subvariety of $W$. But $L_{i} \cup L_{j}$ is fixed pointwise by this intersection. It follows that $\left(\sigma_{i}\right) \cap\left(\sigma_{j}\right)$ keeps each point in a neighborhood of $x$ fixed. This implies that $\left(\sigma_{i}\right) \cap\left(\sigma_{j}\right)$ acts trivially on $W$. This contradicts the assumption that $G$ acts faithfully on $W$.

Since each $\left(\sigma_{i}\right)$ is normal in $G$ and their mutual intersections are trivial it follows that $\sigma_{i}$ commute with each other. This proves that $G_{x}$ is abelian.

We have an interesting consequence of the previous discussion.

Lemma 4. Let $W$ be a smooth projective surface with a morphism $\pi: W \rightarrow D$ onto a smooth projective curve $D$ such that a general fiber of $\pi$ is irreducible. Suppose that a cyclic group $G$ of prime power order $p^{l}$ acts faithfully on $W$ such that the fibers of $\pi$ are permuted by $G$. Let $Z:=W / G$ be the normal projective surface and let $h: Z \rightarrow C$ be the induced surjective morphism onto a smooth projective curve $C$ with connected fibers. Assume further that every fiber of $h$ is irreducible. Then any two ramified curves in $W$ are mutually disjoint. 
In particular, if $\Gamma \subset W$ is a ramified curve for the morphism $W \rightarrow Z$ such that $\pi(\Gamma)=D$ then there is no ramified curve $\Delta \subset W$ for $W \rightarrow Z$ which is contained in a fiber of $\pi$.

Proof. Suppose that this is not true. Let $R_{1}, R_{2}$ be distinct irreducible ramified curves and let $x \in R_{1} \cap R_{2}$. Since $G$ is cyclic of order $p^{l}$ there is a unique subgroup $G_{p}$ of order $p$ in $G$ and it is contained in every non-trivial subgroup of $G$. It follows that $G_{p}$ keeps points in $R_{1} \cup R_{2}$ fixed. This contradicts the fact that the set of fixed points of $G_{p}$ is a smooth subvariety of $W$.

Let $\Delta$ be contained in a fiber $F_{0}$ of $\pi$. Since the fibers of $h$ are irreducible the irreducible components of $F_{0}$ are conjugate under $G$. Thus every irreducible component of $F_{0}$ is ramified for the map $W \rightarrow Z$. Now $\Gamma$ meets at least one irreducible component of $F_{0}$, say $\Delta$. This contradicts the first part above. This proves the second part of the lemma.

Similar proof gives the following result which will be used later.

LEMma 5. With the same notation as in Lemma 4, let $G$ be a finite group whose order is a product of distinct primes. Assume that every isotropy subgroup $G_{x}$ is normal in $G$. If $\Gamma, \Delta$ are as in Lemma 4 which are both ramified for $W \rightarrow Z$ then $\left|G_{\Gamma}\right|,\left|G_{\Delta}\right|$ are relatively coprime.

The proof uses Lemma 3 at any common point of $\Gamma, \Delta$ and the arguments in the proof of Lemma 4 .

Remark. Lemmas 4, 5 will be used later when $Z$ is a $\mathbf{P}^{1}$-bundle.

Closely related to the above results is the following result which is of independent interest. More general results of this nature were proved in [1].

LEMMa 6. Let $G$ be a finite abelian group acting on an irreducible normal algebraic variety $W$ and $Z:=W / G$. Assume that $G$ is generated by the isotropy subgroups $G_{x}$ of points in $W$. Then the natural homomorphism $\pi_{1}(W) \rightarrow \pi_{1}(Z)$ is surjective.

Proof. Let $\Gamma$ be the image of $\pi_{1}(W) \rightarrow \pi_{1}(Z)$. There is a connected covering $Z^{\prime} \rightarrow Z$ such that $\pi_{1}\left(Z^{\prime}\right)=\Gamma$ (as subgroup of $\pi_{1}(Z)$ ). We have a lift $W \rightarrow Z^{\prime}$ of the morphism $\pi: W \rightarrow Z$ such that $Z^{\prime}$ is a quotient $W / H$ for some subgroup $H$ of $G$. Assume that degree $Z^{\prime} \rightarrow Z$ is $>1$. Then $H$ is a proper subgroup of $G$ and $G / H$ acts on $Z^{\prime}$ such that $Z^{\prime} /(G / H)=Z$. Hence there is a point $x \in W$ such that $G_{x}$ is not contained in $H$. Then the image of $x$ in $Z^{\prime}$ has a non-trivial isotropy subgroup contained in $G / H$. This is a contradiction since $Z^{\prime} \rightarrow Z$ is a non-trivial covering space.

Now let $X$ be a smooth projective surface, $G$ a finite group of automorphisms of $X$, and let $Y:=X / G$ be the normal projective quotient surface.

For a germ of a singular point $(Y, p)$, the local fundamental group $\pi_{1}(Y-p)$ is finite. Let $x \in X$ be a point lying over $p$. Then $\pi_{1}(Y-p)$ is a quotient of $G_{x}$, and $\left|G_{x}\right||| G \mid$. In fact, if $H_{x}$ is the normal subgroup of $G_{x}$ generated by pseudo-reflections contained in $G_{x}$ then the image of $x$ in $X^{\prime}:=X / H_{x}$ is again a smooth point, say $x^{\prime}$. The group 
$G_{x} / H_{x}$ acts on $X^{\prime}$ and the image of $x^{\prime}$ in $X^{\prime} /\left(G_{x} / H_{x}\right)$ has analytically isomorphic local ring as that of $p$ on $Y$.

By the well-known result of D. Mumford about topology of a normal surface singularity, the absolute value of the determinant of the intersection form of the exceptional divisor at $p$ (called determinant, for simplicity) is $\left|\pi_{1}(Y-p) /\left[\pi_{1}(Y-p), \pi_{1}(Y-p)\right]\right|$. This group is a quotient of $G_{x} /\left[G_{x}, G_{x}\right]$, whose order is a factor of $|G|$.

These observations will be implicitly used later.

Let $\varphi: Y \rightarrow C$ be a morphism on a normal projective surface $Y$ onto a smooth projective curve $C$. Following Sakai $[6]$ it is called a minimal ruled fibration, if a general fiber of $\varphi$ is isomorphic to $\mathbb{P}^{1}$ and every fiber of $\varphi$ is irreducible.

Assume that $\varphi$ is a minimal ruled fibration and $F_{0}$ a scheme-theoretic fiber of $\varphi$. We say that $F_{0}$ is a singular fiber of $\varphi$ if $F_{0}$ contains a singular point of $Y$. The following result proved in $[\mathbf{6}]$ is crucially used in our work.

Proposition 1 (Sakai's result). Let $F_{0}$ be a singular fiber of $\varphi$. We have the following assertions.

(i) Y has at worst rational singular points.

(ii) $F_{0}$ contains at most two singular points of $Y$.

(iii) The multiplicity $m$ of $F_{0}$ is $>1$.

(iv) If $\tau: \widetilde{Y} \rightarrow Y$ is a minimal resolution of singularities of $Y$ then the inverse image $\tau^{*}\left(F_{0}\right)$ has the following dual graph.

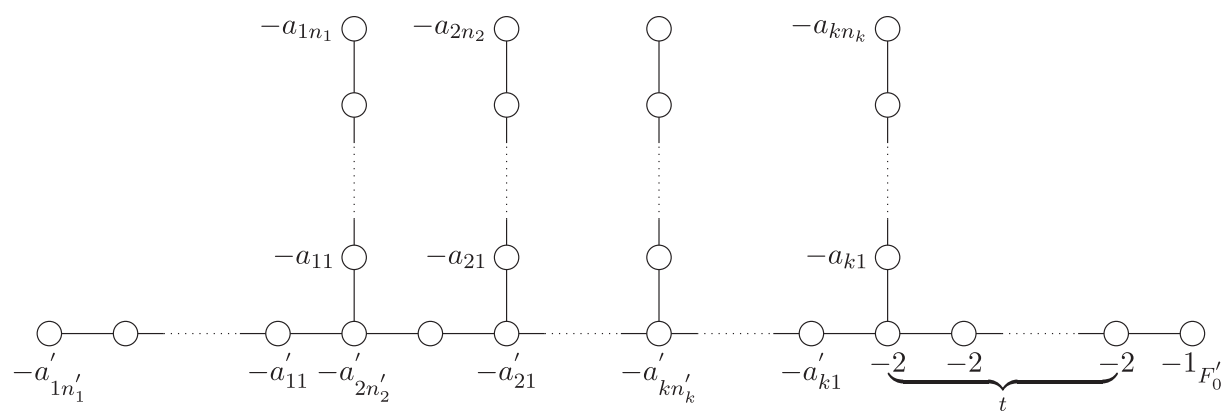

Figure 2.

Here $a_{i j} \geq 2, a_{i j}^{\prime} \geq 2$ for all $i, j \geq 0$. The curve $F_{0}^{\prime}$ is the proper transform of $F_{0}$. We allow $t=0$.

Let $\left[a_{1}, \ldots, a_{n}\right]=a_{1}-\frac{1}{a_{2}-}$ denote a continued fraction where all $a_{i} \geq 2$. Let $\left[a_{1}, \ldots, a_{n}\right]=d / e$, where $d$, e are mutually prime positive integers.

(v) If $\left[a_{11}, \ldots, a_{1 n_{1}}\right]=d_{1} / e_{1}$, then $\left[a_{11}^{\prime}, \ldots, a_{1 n_{1}^{\prime}}^{\prime}\right]=d_{1} /\left(d_{1}-e_{1}\right)$ and for $i \geq 2$, if $\left[a_{i 1}, \ldots, a_{i n_{i}}\right]=d_{i} / e_{i}$ then $\left[a_{i 1}^{\prime}, \ldots, a_{i n_{i}^{\prime}-1}^{\prime}, a_{i n_{i}}^{\prime}-1\right]=d_{i} /\left(d_{i}-e_{i}\right)$. 
(vi) The multiplicity of $F_{0}$ is equal to $\prod_{i=1}^{k} d_{i}$.

(vii) $Y$ contains at most one irreducible curve $C_{0}$ with $C_{0}^{2}<0$. If such a curve $C_{0}$ exists then for any other horizontal irreducible curve $D_{0}$ on $Y$ we have $D_{0}^{2}>0$.

REMARK. We will apply (vii) above as follows. Let $Y=X / G$ and $B_{0}$ be the branch divisor in $Y$-Sing $Y$. If $B_{0}$ is not irreducible then $B_{i}^{2}>0$ for some component of $B_{0}$. In this case we can apply the Nori's theorem (see Section 5).

\section{The case when $X / G$ is a $\mathbb{P}^{1}$-bundle.}

In this section we begin with a more general finite group, and not just those whose order is a product of distinct primes, as it is in the main theorem of this article. Let $X$ be a smooth projective surface, $G$ a finite group acting faithfully on $X$ and $Y:=X / G$ the quotient surface. Assume that $\varphi: Y \rightarrow C$ is a surjective morphism onto a smooth curve such that all the fibers of $\varphi$ are irreducible. There is an induced morphism $X \rightarrow C$. By taking Stein factorization we get a morphism $\psi: X \rightarrow D$ onto a smooth curve $D$ with an irreducible general fiber. Now $G$ permutes the fibers of $\psi$. Let $\pi: X \rightarrow Y$ be the quotient morphism. If some fiber $G_{0}$ of $\psi$ is reducible then its irreducible components are conjugate under $G$. In particular, they have the same ramification index over their common image which is a fiber of $\varphi$. Any irreducible curve $\Gamma$ in $X$ such that $\psi(\Delta)=D$ meets at least one of the irreducible components of $G_{0}$.

We call such a curve $\Gamma$ a horizontal curve for $\psi$.

We assume that the following conditions are satisfied:

(1) Any isotropy subgroup $G_{x}$ is normal in $G$.

(2) No isotropy subgroup has any isolated fixed point in $X$.

Note. It should be emphasized that the assumption (2) above is appearing only because we are handling arbitrary finite groups. The main theorem of this article, deals with those finite groups $G$, whose order is a product of distinct primes. For these groups, which is the object of our study, the assumption (2) is naturally satisfied. This follows from our subsequent results. But it is useful to discuss the more general setting for clarity.

Let $H_{1}, H_{2}, \ldots$ be the horizontal irreducible ramified curves in $X$. Let $G_{H_{i}}$ be the isotropy subgroup of a general point in $H_{i}$. By assumption (1) $G_{H_{i}}$ is normal in $G$. By Lemma $3, G_{H_{i}}$ is abelian. Let $G_{H}$ be the (normal) subgroup of $G$ generated by all the $G_{H_{i}}$ for $i=1,2, \ldots$. Clearly $G_{H}$ is normal in $G$. Similarly, let $F_{1}, F_{2}, \ldots$ be the fibers of $\psi$ which are ramified over $Y$ and let $G_{F_{j}}$ be the (normal) isotropy subgroup of a general point in $F_{j}$. We say that an irreducible curve $\Delta \subset X$ is vertical for $\psi$ if $\Delta$ is contained in a fiber of $\psi$.

Let $G_{V}$ be the (normal) subgroup of $G$ generated by $G_{F_{j}}$ for $j=1,2, \ldots$

ClaIm. The natural morphism $X /\left(G_{H} \cdot G_{V}\right) \rightarrow Y$ is unramified outside finitely many points. In particular, if $Y$ is smooth then this morphism is unramified. 
For, suppose that $x_{1} \in X /\left(G_{H} \cdot G_{V}\right)$ is a ramified point. Let $x \in X$ be a point lying over $x_{1}$. Then $x$ is ramified for the morphism $X \rightarrow Y$. Since the isotropy subgroup $G_{x}$ fixes a curve passing through $x$ pointwise by the assumption (2), $G_{x} \subset G_{H}$. $G_{V}$. As in Lemma 1 we know that $\left|G_{H_{i}}\right|$ is the ramification index of $H_{i}$ over its image in $Y$. By the same argument $\left|G_{H_{i}}\right|$ is the ramification index of $H_{i}$ over its image in $X /\left(G_{H} \cdot G_{V}\right)$. Now it is clear that no curve in $X /\left(G_{H} \cdot G_{V}\right)$ is ramified over its image in $Y$. If $Y$ is smooth then purity of branch locus implies the second assertion in the claim.

In view of the above observations we can decompose the morphism $X \rightarrow Y$ as $X \rightarrow X_{1} \rightarrow Y$, where $X_{1} \rightarrow Y$ is unramified outside finitely many points. If $Y$ is smooth then $X_{1} \rightarrow Y$ is unramified.

In what follows we will often replace $Y$ by $X_{1}$ so $G=G_{H} \cdot G_{V}$. Now there is no non-trivial decomposition of $X \rightarrow Y$ as $X \rightarrow X^{\prime} \rightarrow Y$ where $X^{\prime} \rightarrow Y$ is unramified outside finitely many points (i.e. $X^{\prime} \rightarrow Y$ is divisorially unramified).

Combining the arguments above we have the following result.

Lemma 7. Let $X, Y=X / G$ be as above. Assume that every isotropy subgroup $G_{x}$ is normal in $G$, and $G=G_{H} \cdot G_{V}$. Then the morphism $X \rightarrow X / G_{H}$ has only horizontal ramified curves and $X / G_{H} \rightarrow Y$ has only vertical ramified curves.

Of particular interest to us is the case when $Y$ is a $\mathbb{P}^{1}$-bundle over $C$. Since $X / G_{H} \rightarrow$ $Y$ has only vertical ramification we see easily that $X / G_{H}$ is also a $\mathbb{P}^{1}$-bundle over a suitable curve $C^{\prime}$. Then $X \rightarrow X / G_{H}$ has only horizontal ramified curves. In view of this, we will often assume that $X \rightarrow Y$ has only horizontal ramification.

(3) From now onwards till the end of this section we will assume that the quotient surface $Y=X / G$ is a $\mathbb{P}^{1}$-bundle $\varphi: Y \rightarrow C$ over a smooth curve $C$, but $X$ does not admit any $C^{\infty}$-fiber bundle structure. Further, $|G|$ is a product of distinct primes. As said in the Introduction we will also assume the Basic Setup stated on the third page of this paper.

REMARK. The motivation for this assumption is that the topology of differentiable fibers bundles over smooth projective surfaces is better understood.

In Section 7 we will give an example to show that even when $G$ is abelian, the results below are not true without the hypothesis in the Main Theorem (see Introduction).

Let $\psi: X \rightarrow D$ be the induced morphism onto a smooth curve $D$ with an irreducible general fiber. Now $G$ permutes the fibers of $\psi$. Let $\pi: X \rightarrow Y$ be the quotient morphism.

The crucial ideas in this section have already been used in [4] and [3]. From results in [5, Chapter 5, Section 2.8.1], there is a suitably normalised locally free sheaf $\mathcal{E}$ of rank 2 on $C$ such that $Y=\mathcal{P}(\mathcal{E})$. Let $g$ be the genus of $C$. Let $E$ be the divisor corresponding to $\wedge^{2} \mathcal{E}, e=-\operatorname{deg} E$ and $C_{0}$ a cross-section of $\varphi$ with $C_{0}^{2}=-e$. Let $l$ be a general fiber of $\varphi$. Every divisor on $Y$ is numerically equivalent to an integer linear combination of $C_{0}$ and $l$.

By Lemma 3 every $G_{x}$ is abelian. This implies by Lemma 2 that the ramification divisor $R$ in $X$ is a simple normal crossing divisor (SNC, for short). Purity of branch locus implies that there are no isolated branch points in $Y$. Write $R=\bigcup R_{i}$, where $R_{i}$ are irreducible components of $R$. Let $B_{i}=\pi\left(R_{i}\right)$. Let $B_{i} \equiv a_{i} C_{0}+b_{i} l$. 
Claim. There is at least one horizontal ramified curve for $X \rightarrow Y$.

If this is not true then the induced morphism on $X$ is a $\mathbb{P}^{1}$-fibration which is actually a bundle. By assumption this cannot happen.

Case 1: We first consider the case when there are no vertical ramified curves.

In this case each $B_{i}$ is horizontal and hence $a_{i}>0$.

Case 1.1: Assume that $e \geq 0$. If $B_{i}$ is different from $C_{0}$ then by Proposition 2.20 on $[\mathbf{5}], a_{i}>0, b_{i} \geq a_{i} e$. Then $B_{i}^{2} \geq 0$ and equality holds only if $e=0, b_{i}=0$. Then $B_{i} \equiv a_{i} C_{0}$.

Case 1.2: Assume that $e<0$. If $B_{i} \neq C_{0}$ then using Proposition 2.21 in [5] we check that $B_{i}^{2}>0$. Thus, in this case each $B_{i}^{2}>0$. By the assertion of Lemma 2 we see that $\bigcup R_{i}$ is SNC and hence so is $\bigcup B_{i}$. By Nori's theorem in Section 5, the natural homomorphism $\pi_{1}(X) \rightarrow \pi_{1}(Y)$ has finite abelian kernel. Clearly, $\pi_{1}(Y) \cong \pi_{1}(C)$. If $F$ is a general fiber of $\psi$ then the composite morphism $F \rightarrow D \rightarrow C$ shows the image of the homomorphism $\pi_{1}(F) \rightarrow \pi_{1}(X)$ is contained in the kernel of $\pi_{1}(X) \rightarrow \pi_{1}(Y)$. This shows that the image of $\pi_{1}(F)$ in $\pi_{1}(X)$ is a finite abelian group.

Now we consider Case 1.1 in more detail. First let $e>0$.

By the above claim there is at least one horizontal ramified curve. The only irreducible curve on $Y$ with self-intersection $<0$ is $C_{0}$ and every other horizontal irreducible curve has strictly positive self-intersection. For any finite morphism $\Delta \rightarrow \mathbf{P}^{1}$ of degree $>1$ at least two points in $\mathbf{P}^{1}$ are branched so it cannot happen that $C_{0}$ is the only branched curve. Let $B_{1}, B_{2}, \ldots$ be the irreducible branch curves with $B_{i}^{2}>0$ for $i=1,2, \ldots, n$. Let $B_{0}=C_{0}$ if $C_{0}$ is ramified. The union $\bigcup_{0}^{n} B_{i}$ is SNC. By Nori's theorem the kernel of the homomorphism $\pi_{1}\left(X-\pi^{-1} C_{0}\right) \rightarrow \pi_{1}\left(Y-C_{0}\right) \cong \pi_{1}(C)$ is finite abelian. Then as in Case 1.2 we deduce that $\pi_{1}(F) \rightarrow \pi_{1}(X)$ has finite abelian image.

Now let $e=0$. Let $B_{1}, B_{2}, \ldots, B_{m}$ be the irreducible branch curves with $B_{i}^{2}=0$ and $B_{j}$ be the branch curves with $B_{j}^{2}>0$ for $j=m+1, \ldots, n$.

For $i=1, \ldots, m$ we have $B_{i} \equiv a_{i} C_{0}$ with $a_{i}>0$. If $g=0$ and $a_{i}>1$ then the linear system $\left|a_{i} C_{0}\right|$ does not have any irreducible member. Hence in this case $a_{i}=1$ and $B_{i}$ is a cross-section disjoint from $C_{0}$. In this case $Y$ is isomorphic to $\mathbb{P}^{1} \times \mathbb{P}^{1}$.

Assume that $g>0$. Using the expression $K_{Y} \equiv-2 C_{0}+(2 g-2) l$, and the genus formula we infer that if $g>0$ then $2 g_{B_{i}}-2=a_{i}(2 g-2)$. By Riemann-Hurwitz formula the morphism $B_{i} \rightarrow C$ is unramified for $i=1,2, \ldots, m$. If $b_{j} \neq 0$ then $B_{j}^{2}>0$.

For $j>m$ we have $B_{j} \equiv a_{j} C_{0}+b_{j} l$ and $b_{j}>0$. Then $B_{j}$ meets every irreducible curve on $Y$. Let $G_{i}$ be the isotropy subgroup of a general point of $R_{i}$ for $i=1, \ldots, n$. By the proof of Lemma 3 for $i \neq j$ the intersection $G_{i} \cap G_{j}=(e)$. Let $G_{0}$ be the normal subgroup of $G$ generated by $G_{1}, G_{2}, \ldots, G_{m}$ and let $G_{0}^{\prime}$ be the normal subgroup generated by $G_{j}$ for $j=m+1, \ldots, n$. Then $G_{0} \cap G_{0}^{\prime}=(e)$ since $|G|$ is a product of distinct primes.

Let $X_{1}:=X / G_{0}^{\prime}$. Then the branch curves for $X_{1} \rightarrow Y$ are $B_{1}, \ldots, B_{m}$. It follows that $X_{1}$ is a differentiable fiber bundle. In particular $X_{1}$ is a smooth surface. The inverse image of $\bigcup_{j=m+1}^{n} B_{j}$ in $X_{1}$ is an SNC divisor which is the branch curve for $X \rightarrow X_{1}$. The inverse image of each $B_{j}$ for $j>m$ splits into disjoint smooth curves in $X_{1}$, and hence the self-intersection of each of these irreducible components is positive. Again by 
Nori's theorem in Section 5, the homomorphism $\pi_{1}(X) \rightarrow \pi_{1}\left(X_{1}\right)$ is an isomorphism. To see the surjectivity of this homomorphism we use Lemma 6 . The injectivity follows from the proof of Nori's theorem in Section 5.

Case 2: We consider the general case when both horizontal and vertical ramification exists.

By Lemma $5, G_{H} \cap G_{V}=(e)$. The morphism $X \rightarrow X / G_{H}$ has only horizontal ramification and $X / G_{H} \rightarrow X /\left(G_{H} \cdot G_{V}\right)$ has only vertical ramification. Finally, the morphism $X /\left(G_{H} \cdot G_{V}\right) \rightarrow X / G$ is unramified. It follows easily that $X / G_{H}$ is a $\mathbb{P}^{1}$-bundle and $X \rightarrow X / G_{H}$ is reduced to Case 1 .

This proves Theorem 1 (Main Theorem) stated in the Introduction for the case $X / G$ is a $\mathbb{P}^{1}$-bundle.

We now state an analogous result of the Main Theorem for smooth surface of general type with an action of any finite cyclic group such that $X / G$ is a $\mathbb{P}^{1}$-bundle.

THEOREM 1'. Let $X$ be a smooth projective surface of general type, $G$ a finite cyclic group acting on $X$ such that $Y:=X / G$ is a $\mathbb{P}^{1}$-bundle. Then we have the following assertions:

(1) $\operatorname{gcd}\left(\left|G_{H}\right|,\left|G_{V}\right|\right)=1$.

(2) With $K:=G_{H} \cdot G_{V}$ the morphism $X / G_{K} \rightarrow X / G$ is finite unramified, so that $X / G_{K}$ is also a $\mathbb{P}^{1}$-bundle.

(3) $X / G_{H}$ is a $\mathbb{P}^{1}$-bundle over a smooth curve $\widetilde{C}$. Letting $F$ to be a general fiber of the induced morphism $X \rightarrow D$ the image of the homomorphism $\pi_{1}(F) \rightarrow \pi_{1}(X)$ is a finite abelian group.

We will omit the proof of the above result.

\section{Fiber multiplicity and $|G|$.}

In this section we will prove the following general result.

TheOREm 2. Let $\psi: X \rightarrow D$ be a fibration on a smooth projective variety $X$ onto a smooth projective curve $D$ with irreducible general fiber. Suppose that $H$ is a finite group of automorphisms of $X$ which permutes the fibers of $\psi$ to fibers of $\psi$. Let $Y:=X / H$ with induced fibration $\varphi: Y \rightarrow C$ with irreducible general fiber. Let $\sigma: \widetilde{Y} \rightarrow Y$ be a resolution of singularities of $Y$ and induced fibration $\widetilde{\varphi}: \widetilde{Y} \rightarrow C$. Let $\widetilde{F}_{0}$ be a fiber of $\widetilde{\varphi}$ and $G_{0}$ a fiber of $\psi$ mapping onto $F_{0}:=\sigma\left(\widetilde{F}_{0}\right)$. Then the multiplicity of $G_{0}$ is at most equal to $|H|$ times the multiplicity of $\widetilde{F}_{0}$.

Proof. We will use the following easy observation.

Let $\tau: X_{1} \rightarrow X$ be the blowing up with center a point in $G_{0}$ and let $G_{1}$ be the scheme-theoretic inverse image of $G_{0}$ in $X_{1}$. Then $\operatorname{mult}\left(G_{1}\right)=\operatorname{mult}\left(G_{0}\right)$.

We will first prove that $\operatorname{mult}\left(G_{0}\right) \leq|H| \cdot \operatorname{mult}\left(F_{0}\right)$.

Let $f: X \rightarrow Y$ be the quotient map. 
Let $m:=\operatorname{mult}\left(F_{0}\right)$. Write $F_{0}=m A_{0}$, where $A_{0}$ has multiplicity 1 . Then $A_{0}=$ $\sum a_{i} A_{i}$, where $A_{i}$ are the irreducible components of $A_{0}$ and $\operatorname{gcd}\left\{a_{1}, \ldots, a_{n}\right\}=1$. Now $f^{*}\left(F_{0}\right)=m \sum a_{i}\left(\sum b_{i} B_{i j}\right)$, where $B_{i j}$ are the irreducible divisors in $X$ lying over $A_{i}$ with ramification index $b_{i}$ (since $f$ is Galois). Clearly $b_{i}|| H \mid$ for each $i$. Let $p:=\psi\left(G_{0}\right), q=$ $\varphi\left(F_{0}\right)$. If $e$ is the ramification index for the map $D \rightarrow C$ at $p$ then we see that the multiplicity of $G_{0}$ is $(m / e)$ times the greatest common divisor of $b_{1}, b_{2}, \ldots$

Now it follows that the multiplicity of $G_{0}$ is at most equal to $m \cdot|H|$.

Let $\bar{X}$ be the normalization of $\widetilde{Y}$ in the function field of $X$. Then there is an induced fibration $\bar{\psi}: \bar{X} \rightarrow D$. Let $\bar{G}_{0}$ be the scheme-theoretic inverse image of $G_{0}$ in $\bar{X}$. The previous argument shows that $\operatorname{mult}\left(\bar{G}_{0}\right) \leq|H| \cdot \operatorname{mult}\left(\widetilde{F}_{0}\right)$. If $\widetilde{X} \rightarrow \bar{X}$ is a resolution of singularities of $\bar{X}$ and $\widetilde{G}_{0}$ the corresponding fiber in $\widetilde{X}$ then $\operatorname{mult}\left(\widetilde{G}_{0}\right)=\operatorname{mult}\left(G_{0}\right)$. But $\widetilde{G}_{0}$ is a union of proper transforms of irreducible components of $\bar{G}_{0}$ and exceptional curves obtained by resolution of singularities. Hence $\operatorname{mult}\left(G_{0}\right)=\operatorname{mult}\left(\widetilde{G}_{0}\right) \leq \operatorname{mult}\left(\bar{G}_{0}\right) \leq$ $|H| \cdot \operatorname{mult}\left(\widetilde{F}_{0}\right)$. This proves the theorem.

We have the following application of this result. This was proved in [3, Theorem 2], by a more complicated argument.

Corollary. Let $X$ be a smooth projective surface with a morphism $\psi: X \rightarrow D$ onto a smooth projective curve $D$ such that a general fiber of $\psi$ is a smooth hyperelliptic curve of genus $g \geq 2$. Then the multiplicity of any fiber of $\pi$ is at most 2 .

Proof. In [3, Lemma 5], it is proved that the group $H:=\mathbf{Z} /(2)$ acts on $X$ such that every fiber of $\psi$ is stable under this action and the action on a smooth fiber is the hyperelliptic involution. Let $Y:=X / H$. Then $Y$ is a normal projective surface with an induced $\mathbf{P}^{1}$-fibration $\varphi: Y \rightarrow C$. Let $\widetilde{Y} \rightarrow Y$ be a resolution of singularities of $Y$ with the induced $\mathbf{P}^{1}$-fibration $\widetilde{\varphi}: \widetilde{Y} \rightarrow C$. It is well-known, and easy to prove, that the multiplicity of any fiber of $\widetilde{\varphi}$ is 1 . Now the result follows immediately from Theorem 2.

\section{Minimal $\mathbb{P}^{1}$-fibrations as quotient.}

Our next result deals with the case when $\varphi: Y=X / G \rightarrow C$ is a minimal ruled fibration. We will assume that the fibration $\psi: X \rightarrow D$ is not a $\mathbb{P}^{1}$-fibration. Let $F_{0}$ be a singular fiber of $\varphi$.

Theorem 3. If $g: Y \rightarrow C$ is a minimal $\mathbb{P}^{1}$-fibration then the multiplicity of any fiber $F_{0}$ divides $|G|$.

Proof. We will use Proposition 1. Clearly, $Y$ has at most quotient singular points. By Proposition 1, $F_{0}$ contains at most two singular points. We will divide the proof according as $F_{0}$ has one or two singular points.

Case 1: $\quad F_{0}$ contains a unique singular point $p$ of $Y$.

In the notation of Proposition 1 , let $\widetilde{F}_{0}$ be the inverse image of $F_{0}$ in $\widetilde{Y}$. Then the dual graph of $\widetilde{F}_{0}$ looks like either Figure 3 or Figure 4 . 


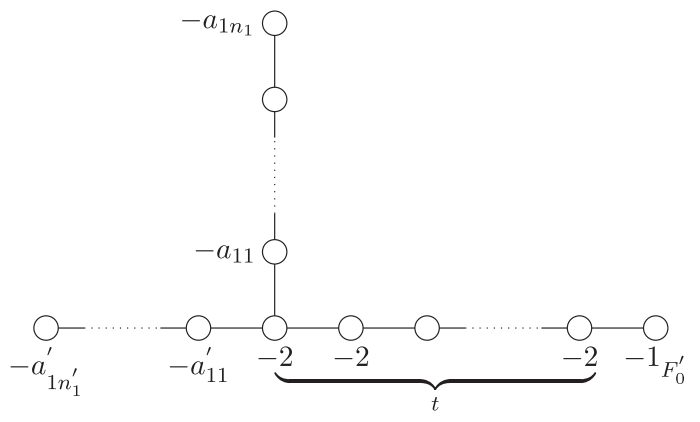

Figure 3.

$F_{0}^{\prime}$ is the proper transform of $F_{0}$ and it is the unique $(-1)$-curve in $\widetilde{F}_{0}$. We have $t \geq 1$. The multiplicity of $F_{0}$ is equal to the determinant of the vertical branch starting with $-a_{11}$ and ending with $-a_{1 n_{1}}$, say $d$. It is easy to check that the determinant of the intersection form of the resolution of singularities of $p$, say $\delta$, is $d^{2}$. The determinant is a factor of $|G|$.

Thus, (multiplicity $\left.F_{0}\right)^{2}$ divides $|G|$.

The following case can also occur.



Figure 4 .

The number of $(-2)$ curves on the right most branch in the above diagram is $t-1$. In this case, $4 t$ divides $|G|$ and the multiplicity of $F_{0}$ is $2 t$. Hence, multiplicity of $F_{0}$ divides $|G|$.

Case 2: Suppose $F_{0}$ contains two singular points of $Y$.

Using Proposition 1, we see that the dual graph of $\widetilde{F}_{0}$ is one of the following cases (A) or (B).

(A)

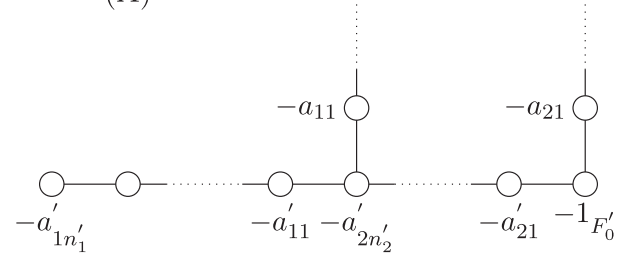

(B)<smiles>OCOO</smiles><smiles>C1CCOC1</smiles><smiles>COOC1(OC)OCCO1</smiles>

Figure 5 . 
It follows that at least one of the singular points is a cyclic quotient singular point.

Subcase (A): Let $d_{1}$ be the determinant of the first vertical branch starting with $-a_{11}$, and let $d_{2}$ be the determinant of the second vertical branch starting with $-a_{21}$. By Proposition 1 , if $\left[a_{21}, \ldots, a_{2 n_{2}}\right]=d_{2} / e_{2}$, then $\left[a_{21}^{\prime}, \ldots, a_{2 n_{2}^{\prime}-1}^{\prime}, a_{2 n_{2}^{\prime}}^{\prime}-1\right]=d_{2} /\left(d_{2}-e_{2}\right)$. The multiplicity of $F_{0}$ is $d_{1} \cdot d_{2}$. Now $d_{1}=2$ or 3 since $Y$ has only quotient singular points. If $d_{1}=2$ then the dual graph of $\widetilde{F}_{0}$ is

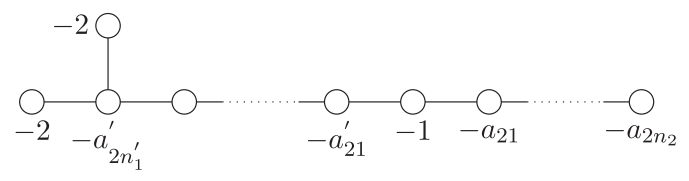

Figure 6.

If $d_{1}=3$ then by Proposition 1 we see that the dual graph of $\widetilde{F}_{0}$ is

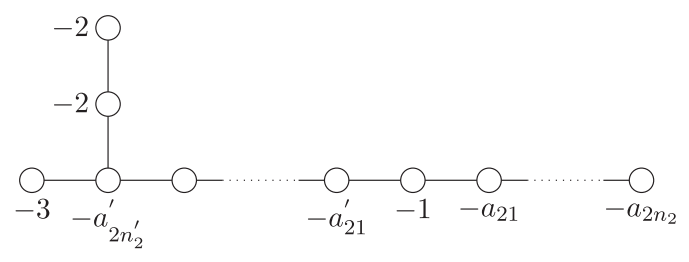

Figure 7.

In case $d_{1}=2$, we check easily that the determinant of the non-cyclic singularity is $4 \cdot d_{2}$. Now multiplicity $m$ of $F_{0}$ is $2 \cdot d_{2}$. Since $4 d_{2}|| G \mid$, we see that $m|| G \mid$.

Suppose $d_{1}=3$. Then we check that the determinant of the non-cyclic singularity is $9 \cdot d_{2}$, which divides $|G|$. Multiplicity $m$ of $F_{0}$ is $3 \cdot d_{2}$. Thus $m|| G \mid$. We have also the case

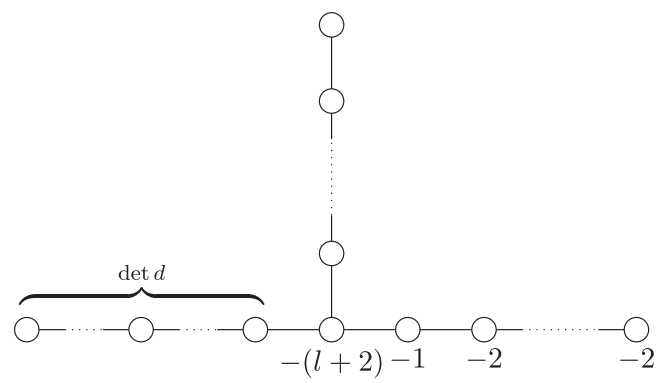

Figure 8 .

The number of $(-2)$ curves on the right most branch is $l$. Then $m=d(l+1)$, $\delta=d^{2}(l+1$ ) (where $\delta$ is the absolute value of the determinant of the intersection form of the resolution of singularities). We note for future use the following observation.

For the Subcase (A), $|G|$ is divisible by $l^{2}$ for some prime number $l$. 
Corollary. Assume that $|G|$ is a product of distinct primes. Then Subcase (A) cannot occur on any singular fiber of $Y \rightarrow C$.

Subcase (B): In this case, if $\left[a_{11}, \ldots, a_{1 n_{1}}\right]=d_{1} / e_{1}$ then $\left[a_{11}^{\prime}, \ldots, a_{1 n_{1}^{\prime}}^{\prime}\right]=d_{1} /\left(d_{1}-\right.$ $\left.e_{1}\right)$. The multiplicity $m$ of $F_{0}$ is $d_{1}$. It follows that $m|| G \mid$. This completes the proof of Theorem 3.

If $|G|$ is a product of distinct primes then our proof shows that every singular fiber $F_{0}$ of $Y \rightarrow C$ has two cyclic quotient singular points of $Y$. The dual graph of $\widetilde{F}_{0}$ is a linear chain such that $F_{0}^{\prime}$ is the only $(-1)$-curve in $\widetilde{F}_{0}$ and the multiplicity of $F_{0}$ is equal to the determinant of either of the two singularities. This observation will be important for the proof of Theorem 1.

Proof of Theorem 1 (Main Theorem). Let $X$ be a smooth projective surface with an action of a finite group $G$ such that $|G|$ is a product of distinct primes and all the isotropy subgroups $G_{x}$ are normal in $G$. Assume that $Y:=X / G$ admits a minimal $\mathbb{P}^{1}$-fibration $\varphi: Y \rightarrow C$. Let $\pi: X \rightarrow Y:=X / G$ be the quotient morphism. Assume that the induced fibration $\psi: X \rightarrow D$ is not an essentially $C^{\infty}$-fiber bundle.

For the proof of this result we need a result due to Madhav Nori (see [7]). We state a special case which is enough for our use.

NORI'S THEOREM. Let $\Delta$ and $S$ be reduced curves on a smooth projective surface $Z$ with $\Delta \cup S$ an SNC curve, with $\Delta_{i}^{2}>0$ for every irreducible component of $\Delta$. Let $W$ be a normal projective surface with a finite morphism $\pi: W \rightarrow Z$ which is unramified outside $\Delta \cup S$. Then the kernel of the map $\pi_{1}\left(W-\pi^{-1} S\right) \rightarrow \pi_{1}(Z-S)$ is a finite abelian group.

Let $B_{0}$ be the divisional part of the branch locus for $\pi$. We will first consider the case when $C$ is non-rational. Let $m_{1} G_{1}, m_{2} G_{2}, \ldots, m_{r} G_{r}$ be the singular fibers of $\varphi$. If $Y$ is smooth then the result is proved in [3]. Now assume that $r \geq 1$.

By the solution of Fenchel's Conjecture due to Nielsen-Bundgaard and Fox (see [2]), there exists a finite Galois ramified morphism $\tau: \widetilde{C} \rightarrow C$ such that $\tau$ is ramified precisely over the points $\varphi\left(G_{i}\right)$ with ramification index $m_{i}$.

Let $\widetilde{Y}$ be the normalisation of the fiber product $Y \times_{C} \widetilde{C}$.

Claim. $\widetilde{Y}$ is smooth and the fibers of the morphism $\widetilde{Y} \rightarrow \widetilde{C}$ are irreducible, i.e. $\widetilde{Y}$ is a $\mathbb{P}^{1}$-bundle over $\widetilde{C}$. Further, $\widetilde{Y} \rightarrow Y$ is unramified outside Sing $Y$.

We will only indicate the proof since this must be well-known to experts.

The proof is local with respect to $C$, so we will assume that $\varphi: U \rightarrow D_{1}$ is a $\mathbb{P}^{1}$-fibration on a normal surface $U$ with irreducible fibers and $D_{1}$ is a small disc in $\mathbf{C}$ around the origin. Let the central fiber $F_{0}$ have multiplicity $m$. Assume that $F_{0}$ contains exactly two cyclic quotient singular points $p, q$. Let $U^{\prime} \rightarrow U$ be the minimal resolution of singularities of $U$. The inverse of $F_{0}$ in $U^{\prime}$, say $\widetilde{F}_{0}$, is a linear chain of smooth rational curves and the proper transform $F_{0}$ in $U^{\prime}$ is the unique $(-1)$-curve in $\widetilde{F}_{0}$.

Consider the map $D_{2} \rightarrow D_{1}$ given map $z \rightarrow z^{m}$. Here $D_{2}$ is another copy of $D_{1}, z$ the local parameter on $D_{2}$. Let $\widetilde{U}$ be the normalization of the fiber product $U \times{ }_{D_{1}} D_{2}$. The inverse image of $F_{0}-\{p, q\}$ splits into a certain number of disjoint curves isomorphic 
to $\mathbf{C}^{*}$. The closure of each of these irreducible curves in $\widetilde{U}$ contains two more points. The inverse image of $F_{0}$ in $\widetilde{U}$ is simply-connected. From these we deduce that the inverse image of $F_{0}-\{p, q\}$ in $\widetilde{U}$ is irreducible, it occurs with multiplicity 1 in the $\mathbb{P}^{1}$-fibration on $\widetilde{U}$, and it is a connected covering of $F_{0}-\{p, q\}$ of degree $m$. This implies that the inverse image of $p$ and $q$ are single smooth points.

This shows that $\widetilde{U}$ is a $\mathbb{P}^{1}$-bundle over $D_{2}$.

Let $\widetilde{X}=\overline{X \times_{Y} \widetilde{Y}}$ be the normalisation of the fiber product. We can apply the proof of Theorem 1 in $[\mathbf{3}]$ to the morphism $\widetilde{X} \rightarrow \widetilde{Y}$ without any changes.

The map $\widetilde{X} \rightarrow X$ is unramified. By the proof of Theorem 1 in $[\mathbf{3}]$, the map $\pi_{1}(\tilde{X}) \rightarrow$ $\pi_{1}(\widetilde{Y})$ is an isomorphism.

The map $\pi_{1}\left(\widetilde{Y}^{0}\right) \rightarrow \pi_{1}\left(Y^{0}\right)$ is an injection and $\pi_{1}(\widetilde{X}) \rightarrow \pi_{1}(X)$ is an injection. Hence the composite map $\pi_{1}(\widetilde{X}) \rightarrow \pi_{1}\left(Y^{0}\right)$ is an injection. If $K$ is the kernel of the map $\pi_{1}\left(X^{0}\right) \rightarrow \pi_{1}\left(Y^{0}\right)$, then $K \cap \pi_{1}(\widetilde{X})=(1)$. Hence $K$ injects into the quotient $\pi_{1}(X) / \pi_{1}(\tilde{X})$. (Note that $\widetilde{X} \rightarrow X$ is a Galois morphism so that $\pi_{1}(\tilde{X})$ is a normal subgroup of $\pi_{1}(X)$.) It follows that $K$ is finite.

As in [3], we prove that the universal cover of $X$ is holomorphically convex.

Let $\widetilde{m}_{1} \widetilde{F}_{1}, \ldots, \widetilde{m}_{s} \widetilde{F}_{s}$, be the multiple fibers of $X \rightarrow D$. We have the exact sequence $(\mathrm{X}): \pi_{1}(F) \rightarrow \pi_{1}(X) \rightarrow \Gamma \rightarrow(1)$, where

$$
\Gamma=\left\langle a_{1}, b_{1}, \ldots, a_{g}, b_{g}, e_{1}, \ldots, e_{s} \mid\left[a_{1}, b_{1}\right] \cdots\left[a_{g}, b_{g}\right] e_{1} \cdots e_{s}=1=e_{1}^{\widetilde{m}_{1}}=\cdots=e_{s}^{\widetilde{m}_{s}}\right\rangle .
$$

The image of the composite map $\pi_{1}(F) \rightarrow \pi_{1}(X) \rightarrow \pi_{1}\left(Y^{0}\right)$ factors through $\pi_{1}\left(\mathbb{P}^{1}\right), \mathbb{P}^{1}$ being a general fiber of $Y \rightarrow C$ (image of $F$ in $Y$ ). Hence the image $\pi_{1}(F) \rightarrow \pi_{1}\left(Y^{0}\right)$ is trivial. If the image $\pi_{1}(F) \rightarrow \pi_{1}(X)$ is infinite then kernel $\pi_{1}(X) \rightarrow \pi_{1}\left(Y^{0}\right)$ will be infinite, a contradiction. Hence image $\pi_{1}(F) \rightarrow \pi_{1}(X)$ is finite.

This completes the proof of Theorem 1 in case $C$ is non-rational. Now assume that $C$ is rational. Let $q_{1}, q_{2}, q_{3}$ be three general points in $C$. Let $\widetilde{C} \rightarrow C$ be a Galois ramified cover which is branched only over $q_{1}, q_{2}, q_{3}$ with ramification index $>2$ for each $q_{i}$. Then $\widetilde{C}$ is non-rational. Let $\widetilde{Y}=Y \times_{C} \widetilde{C}$ be the fiber product and let $\widetilde{X}=X \times_{Y} \widetilde{Y}$ be the fiber product. Then $G$ acts on $\widetilde{X}$ such that $\widetilde{Y}$ is the quotient. The $\mathbb{P}^{1}$-fibration on $\tilde{Y}$ is a minimal ruled fibration. Further, $\widetilde{X}$ is smooth.

By the previous case, the image $\pi_{1}(\widetilde{F}) \rightarrow \pi_{1}(\widetilde{X})$ is finite, where $\widetilde{F}$ is a general fiber of the induced fibration $\widetilde{X} \rightarrow \widetilde{C}$. This implies that $\pi_{1}(F) \rightarrow \pi_{1}(X)$ is also finite since $\widetilde{F} \rightarrow F$ is a isomorphism. This completes the proof of Theorem 1 .

\section{The case when $\mathrm{X} / \mathrm{G}$ is a smooth surface of Kodaira dimension zero or an anticanonical rational surface.}

In this section we assume that $G$ has prime order and acts on a smooth projective surface $X$ of general type such that $Y:=X / G$ is either a minimal surface of Kodaira dimension zero or an anticanonical rational surface (that is a smooth rational surface with an effective anticanonical class).

THEOREM 4. Let a group $G$ of prime order act on a smooth projective surface $X$ of general type. Let $X / G$ be either a smooth rational surface with an effective anticanonical 
divisor or a minimal surface of Kodaira dimension zero. Then $\pi_{1}(X)$ is isomorphic to $\pi_{1}(X / G)$. In particular, if $X / G$ is either an anticanonical rational surface or a $\mathrm{K} 3$ surface, then $X$ is simply-connected.

Proof. We first prove the harder case of $X / G$ an anticanonical rational surface. Let $B_{1}, B_{2}, \ldots$ be the irreducible branch divisors in $Y=X / G$. By purity of branch locus there are no isolated branch points. Since $G$ has prime order $B_{i} \cap B_{j}=\emptyset$ for $i \neq j$ and each $B_{i}$ is smooth.

Claim $1 . \quad B_{i}^{2}>0$ for at least one $i$.

Assume that $B_{j}^{2} \leq 0$ for each branch curve. First we have the following.

Claim 2. $\quad-K_{Y} \cdot \Delta \geq 0$ for every irreducible curve $\Delta$ on $Y$.

Suppose the claim is not true. Write $\left|-K_{Y}\right|=a_{1} \Delta_{1}+a_{2} \Delta_{2}+\cdots+a_{r} \Delta_{r}+M$, where $\sum a_{i} \Delta_{i}$ is contained in the fixed part with $-K \cdot \Delta_{i}<0$ for all $i \leq r, M$ does not contain any $\Delta_{i}$ for $i \leq r$, and all $\Delta_{i}^{2}<0$ for $i \leq r$. Then $K_{Y}=-\sum a_{i} \Delta_{i}-M$.

Let $\pi: X \rightarrow Y$ be the quotient morphism. Write $\pi^{*} B_{j}=n_{j} R_{j}$ where $n_{j}$ is the ramification index over $B_{j}$. Hence

$$
\begin{aligned}
K_{X} & =\pi^{*} K_{Y}+\sum\left(n_{i}-1\right) R_{j} \\
& =\pi^{*}\left(K_{Y}+\sum \frac{n_{j}-1}{n_{j}} B_{j}\right) \\
& =\pi^{*}\left(-\sum a_{i} \Delta_{i}-M+\sum\left(\frac{n_{j}-1}{n_{j}}\right) B_{j}\right) .
\end{aligned}
$$

All $B_{j}^{2} \leq 0$ and for $i \leq r, \Delta_{i}^{2}<0$. Then we can see that $\left|N K_{X}\right|$ cannot grow quadratically with $N$. This contradiction shows that $-K_{Y} \cdot \Delta \geq 0$ for all irreducible curves $\Delta$ on $Y$. This proves Claim 2 .

From Claim 2 and the effectivity of $-K_{Y}$ we infer that $K_{Y}^{2} \geq 0$. We fix one $B_{i}$. From $B_{i}^{2} \leq 0, K_{Y} \cdot B_{i} \leq 0$, we have the following cases.

(a) $B_{i}^{2}=-2, K_{Y} \cdot B_{i}=0$. Then $B_{i}$ is a (-2)-curve.

(b) $B_{i}^{2}=0, K_{Y} \cdot B_{i}=-2$. Then $\left|B_{i}\right|$ gives a $\mathbb{P}^{1}$-fibration. In this case all other $B_{j}$ are in fibers of this fibration. Then $X$ admits a $\mathbb{P}^{1}$-fibration, a contradiction.

(c) $B_{i}^{2}=-1=K_{Y} \cdot B_{i}$. Then $B_{i}$ is a $(-1)$-curve, contradicting purity of branch locus.

(d) $B_{i}^{2}=0, K \cdot B_{i}=0$.

Thus, only (a) and (d) are possible. We will first prove:

Claim 3. We can assume that $X$ is minimal.

This is a general assertion and it does not use the assumption after Claim 1.

For, any two $(-1)$-curves on $X$ are disjoint since $X$ is of general type. Hence $G$ keeps the disjoint union of all $(-1)$-curves on $X$ stable. By contracting all the $(-1)$-curves, 
we get a new smooth surface $X_{1}$ with a $G$-action. The surface $Y_{1}=X_{1} / G$ also satisfies $-K_{Y_{1}}$ is effective, since we have a birational morphism $Y \rightarrow Y_{1}$. Continuing this way, we can assume that $X$ is minimal. This proves Claim 3 .

As noted above only (a) and (d) are possible. Note also that $K_{Y}^{2} \geq 0$. If there is a $B_{i}$ such that (d) happens, then by Hodge Index Theorem, we have $K_{Y}^{2}=0$, since $B_{i}$ is not homologous to zero. By Ramification formula we have $K_{X}=\pi^{*}\left(K_{Y}+\right.$ $\left.\sum\left(\left(n_{i}-1\right) / n_{i}\right) B_{i}\right)$, so if at least one of the $B_{i}$ satisfies (d), other $B_{j}$ 's satisfy (a) (or (d) or both), and this implies $K_{X}^{2} \leq 0$. But $X$ was assumed to be a minimal surface of general type, this is a contradiction. So no $B_{i}$ can satisfy (d). This leaves us with (a) for all $B_{i}$. We will show that this cannot happen either. Assume that there is a $B_{i}$ such that $B_{i}^{2}=-2$, then consider $\pi^{*}\left(B_{i}\right)$, this is a curve $C$ in $X$, since $\pi$ is a $p$-fold cyclic cover where $p$ is a prime, it is totally ramified, so $C$ is of the form $p R_{i}$. But since $B_{i}^{2}=-2$ and $X$ is smooth, this forces $p=2$. But then $R_{i}^{2}=-1$, this contradicts the fact that $X$ was minimal. This shows that neither (a) or (d) can occur. The above argument also shows that no $(-2)$-curve can be a branch divisor.

This proves that $B_{i}^{2}>0$ for some $i$, say $i=1$. This proves Claim 1 .

For $i>1$ from $B_{1}$. $B_{i}=0$ using Hodge Index Theorem we get that $B_{i}^{2}<0$ for $i>1$. But $K_{Y} \cdot B_{i} \leq 0$ implies that $B_{i}$ is a $(-2)$-curve for $i>1$. But it has been shown above that no $(-2)$-curve can be in the branch divisor. This shows that $B_{1}$ is the only branch curve in $Y$. Now we can use the proof of Nori's theorem. This shows that $\pi_{1}(X) \rightarrow \pi_{1}(Y)$ is an isomorphism. Finally, this means that $X$ is simply-connected since $Y$ is a smooth projective rational surface.

We now prove the result when $X / G$ is a minimal surface of Kodaira dimension zero. Since $G$ is a group of prime order, the branch divisors of the finite Galois cover corresponding to $X \longrightarrow X / G$ are disjoint union of smooth irreducible curves $B_{i}$. Note that $X$ is a smooth surface of general type, so $K_{X}$ is nef and big. It is assumed that $X / G$ has Kodaira dimension zero, so $K_{X / G}$ is numerically trivial. Hence the canonical bundle formula for finite cyclic covers, says that there has to be at least one $B_{i}$ such that $B_{i}^{2}>0$. Since $B_{i}$ 's are disjoint, and at least one of them has positive self intersection, Nori's Theorem shows that $\pi_{1}(X) \rightarrow \pi_{1}(X / G)$ is an isomorphism.

In particular if $X / G$ is a K3 surface, then $X$ is simply connected. This completes the proof of Theorem 4 .

\section{Examples.}

In this section we give few examples which illustrate how our results in this paper are optimal.

(1) The following is a classical example:

Let $x_{1}, x_{2}, x_{3}$ be coordinates on $\mathbb{C}^{3}$. Let the symmetric group $G:=S_{3}$ act on the $x_{i}$ 's by permutations. The hyperplane $H:=\left\{x_{1}+x_{2}+x_{3}=0\right\}$ is stable under $G$. We can take $x_{1}, x_{2}$ as coordinates on $\mathbb{C}^{2}$. Let $\tau_{i}$ be the three transpositions in $G$, and let $\sigma$ be the 3 -cycle sending $\left(x_{1}, x_{2}, x_{3}\right)$ to $\left(x_{2}, x_{3}, x_{1}\right)$. Each $\tau_{i}$ is a reflection. The three lines $R_{i}$ fixed pointwise by the $\tau_{i}$ (in some order) are $x_{1}=x_{2}, x_{1}+2 x_{2}=$ $0,2 x_{1}+x_{2}=0$. Also, $\sigma$ fixes only the point $(0,0)$. 
The ramification divisor in $H$ is the union of the lines $R_{i}$, which does not have normal crossings. Only $\sigma$ generates a normal subgroup of $G$. The ring of invariants under $\sigma$ is generated by $y_{1}^{3}, y_{2}^{3}, y_{1} \cdot y_{2}$, where $y_{1}=x_{1}-\omega x_{2}, y_{2}=x_{1}-\omega^{2} x_{2}$. Here $\omega$ is a primitive root of unity.

The image of $\tau_{1}$ generates the quotient $G /(\sigma)$. We check easily that the ring of invariants for this action is generated by $y_{1}^{3}-y_{2}^{3}, y_{1}^{3} \cdot y_{2}^{3}$. Thus, $H / G \cong \mathbb{C}^{2}$. The image of the line $x_{1}=x_{2}$ is a curve with equation $z_{2}=a z_{1}^{2}$ for some non-zero constant $z$. Similarly, the images of the other two lines have equations $z_{2}=a^{\prime} z_{1}^{2}, z_{2}=a^{\prime \prime} z_{1}^{2}$ with distinct non-zero constants $a, a^{\prime}, a^{\prime \prime}$. The union of three curves in the branch divisor in $H / G$. Clearly, all these curves are smooth and tangential to each other at $(0,0)$.

(2) This example was conveyed to the second author by Catanese (see also [4]). If $X / G$ is not a $\mathbb{P}^{1}$-fibration, then the image of $\pi_{1}(F) \rightarrow \pi_{1}(X)$ need not be finite. Consider a double cover $X$ of $E \times \mathbb{P}^{1}$ with $E$ an elliptic curve, branched along a smooth bi-section, then $X$ is a genus two fibration. The image of the above map is $\mathbb{Z} \times \mathbb{Z}$ in this case. In this example, $X / G$ has an elliptic fibration with irreducible fibers, but not all fibers of $X$ are irreducible.

(3) If $X / G$ is a $\mathbb{P}^{1}$-fibration and $|G|$ is not a product of primes, the above image can be infinite. This example appears in [4]. This is a Galois cover of $\mathbb{P}^{1} \times \mathbb{P}^{1} . X$ is fiber product of two double covers branched along the smooth divisors $D_{1}$ linearly equivalent to $(2 m+2) f$ and $D_{2}$ linearly equivalent to $\left(6 C_{0}+2 f\right)$, where $C_{0}$ is the minimal cross-section and $f$ is a fiber of $\mathbb{P}^{1} \times \mathbb{P}^{1}$. The group $G$ in this case is $\mathbb{Z} /(2) \times \mathbb{Z} /(2)$. Note that $X$ is fibered over $\mathbb{P}^{1}$ by curves of genus 3 and can be made to have arbitrarily large irregularity, so the image $\pi_{1}(F) \rightarrow \pi_{1}(X)$ cannot be finite.

(4) The factorization theorem is a important step in the proof of the Main Theorem, the fact that $|G|$ is product of distinct primes is used crucially there. The fact that $X$ is smooth is also crucially used in the proofs of the Main Theorem and in Theorem $1^{\prime}$ in Section 3. The following example illustrates these crucial points; it shows these are necessary hypothesis. There are families of examples in $[4]$ where $X / G$ corresponds to the Hirzebruch surfaces and the group is $G=\mathbb{Z}_{4}$. We will specialize to the case when $X / G=\mathbb{P}^{1} \times \mathbb{P}^{1}$ below, for this serves our purpose. Here the respective branch curves are horizontal. Still the finiteness of the $\pi_{1}(F) \rightarrow \pi_{1}(X)$ is not true.

This example is a quadruple Galois cover of $\mathbb{P}^{1} \times \mathbb{P}^{1}$ with $G=\mathbb{Z}_{4}$. Here $X$ is obtained as a composition of two double covers $\varphi_{2}: X \longrightarrow X_{1}$ and $\varphi_{1}: X_{1} \longrightarrow Y$, where $Y=\mathbb{P}^{1} \times \mathbb{P}^{1}$. Here $\varphi_{1}$ is branched along a smooth divisor $D_{2}$ linearly equivalent to $4 C_{0}, \varphi_{2}$ is branched along the ramification of $\varphi_{1}$ and $\varphi_{1}^{*} D_{1}$, where the divisor $D_{1}$ is a smooth divisor linearly equivalent to $(2 m+4) f$. Note that $X$ is fibered over $\mathbb{P}^{1}$ by curves of genus 3 and has irregularity 1 . In this case $X$ is never smooth, and the mildest possible singularities of $X$ are $A_{1}$ singularities.

(5) Another example in [4] serves the following purpose: 
Here $X / G$ is a $\mathbb{P}^{1}$-fibration. The surface $X$ is a bidouble cover branched along a smooth divisor $D_{1}$ linearly equivalent to $2 C_{0}+(2 m+4) f$ and a smooth divisor $D_{2}$ linearly equivalent to $4 C_{0}$. Both branch divisors are horizontal, yet the relevant image is not finite. The group $G$ is $\mathbb{Z} /(2) \times \mathbb{Z} /(2)$. The fact that the $|G|$ is a product of distinct primes in the Main theorem is a necessary one.

\section{References}

[1] M. A. Armstrong, Calculating $\pi_{1}$ of an orbit space, Proc. Amer. Math. Soc., 84 (1982), $267-271$.

[2] T. C. Chau, A note concerning Fox's paper on Fenchel's conjecture, Proc. Amer. Math. Soc., 88 (1983), 584-586.

[ 3 ] R. V. Gurjar, S. Paul and B. P. Purnaprajna, On the fundamental group of hyperelliptic fibrations and some applications, Inventiones Math., 186 (2011), 237-254.

[4] F. J. Gallego and B. P. Purnaprajna, Classification of quadruple Galois covers I, Trans. Amer. Math. Soc., 360 (2008), 5489-5507.

[5] R. Hartshorne, Algebraic Geometry, Graduate Texts in Mathematics, Springer Verlag, 1977.

[6] F. Sakai, Ruled fibrations on normal surfaces, J. Math. Soc. Japan, 40 (1988), 249-269.

[ 7 ] M. Nori, Zariski's conjecture and related problems, Ann. Sci. École Norm Sup. (4), 16 (1983), 305-344.

[8] G. Xiao, $\pi_{1}$ of elliptic and hyperelliptic surfaces, Inter. J. Math., 2 (1991), 599-625.

[ 9 ] G. Xiao, Surfaces fibrées en courbes de genre deux, Lecture Notes in Math., 1137, Springer, Berlin, 1985 .

Rajendra Vasant GuRJAR

School of Mathematics

Tata Institute of Fundamental Research

Homi-Bhabha Road

Mumbai 400005, India

E-mail: gurjar@math.tifr.res.in

Current address:

Department of Mathematics

Indian Institute of Technology Bombay

Powai

Mumbai 400076, India

E-mail: gurjar@math.iitb.ac.in
Bangere P. Purnaprajna

Department of Mathematics

University of Kansas

Lawrence

KS 66045, USA

E-mail: purna@ku.edu 